\title{
Time-resolved observation of ballistic acceleration of electrons in GaAs quantum wells
}

\author{
W Shat, T B Norrist, W J Schaff $\ddagger$ and K E Meyer§ \\ tUltrafast Science Laboratory, University of Michigan, 2200 Bonisteel, Ann Arbor, \\ MI 48109-2099, USA \\ $\ddagger$ The School of Electrical Engineering, Cornell University, Ithaca, NY 14850, USA \\ $\$$ Cavendish Laboratory, Cambridge University, Cambridge, UK
}

\begin{abstract}
We have studied high-field parallel transport of photoinjected carriers in GaAs quantum wells using femtosecond optical spectroscopy. We have directly observed the transient nonequilibrium distribution functions that occur during the field-induced acceleration of the electrons. At a high field $\left(16 \mathrm{kV} \mathrm{cm}^{-1}\right)$, a nonthermal high-energy tail in the distribution function is apparent during the first $150 \mathrm{fs}$, which is due to electrons ballistically accelerated from the band edge. At later times, we observe the relaxation of the applied electric field due both to radiation from the accelerating carriers and from the build-up of space charge as the electron and hole gases separate.
\end{abstract}

Ultrafast time-resolved optical techniques have been used for some time to study relaxation dynamics of hot carriers in semiconductors [1]. These kinds of experiments have been modelled with calculations of the carrier distributions using Monte Carlo methods [2,3]. Monte Carlo calculations have also been extensively used to calculate time-dependent carrier velocities in external electric fields. To gur knowledge, however, experimental studies that directly probe transient carrier distributions in high-field transport have not been performed. We have used femtosecond transient absorption spectroscopy to study parallel transport of photoinjected carriers in GaAs quantum wells, and have observed at high fields the ballistic acceleration of electrons on a $150 \mathrm{fs}$ time scale. Our experiments, which probe the transport on an ultrashort time scale, are thus a complement to studies of ballistic drift in a field-free region using hot-electron transistors, which probe the transport on a very short distance scale $[4,5]$.

In this experiment, an undoped 40-period $50 \AA \mathrm{GaAs} / 150 \AA \mathrm{Al}_{0.3} \mathrm{Ga}_{0.7}$ As quantum well (Qw) structure was used. A horizontal $\mathrm{p}$-i-n diode was fabricated on top of the layer structure to provide an electric field in the plane of the wells. The $\mathrm{p}$ and $\mathrm{n}$ contact electrodes were separated by $50 \mu \mathrm{m}$. Electroabsorption measurements with $10 \mu \mathrm{m}$ spatial resolution across the gap region showed the electric field to be quite uniform across the gap.

The laser source used to perform the experiments produced $100 \mathrm{fs} 620 \mathrm{~nm}$ pulses with $10 \mu \mathrm{J}$ energy at a $2 \mathrm{kHz}$ repetition rate [6]. These pulses were used to generate a white-light continuum, from which the pump (of $10 \mathrm{~nm}$ width centred at $825 \mathrm{~nm}$ ) and probe $(720-$ $840 \mathrm{~nm}$ ) beams were selected. The pump beam was amplified to produce sheet carrier densities of about 2$3 \times 10^{11} \mathrm{~cm}^{-2}$. Dispersion in the probe beam was compensated by prisms so that the absorption spectrum could be obtained with sub-100 fs resolution. Differential spectra were acquired on an OMA (optical multichannel analyser). All experiments were performed at room temperature. The pump wavelength was chosen to resonantly generate heavy-hole excitons in the Qws, in order to start with a cold initial distribution (i.e. the initial kinetic energy of the carriers is zero). The probe pulse then monitored the sequential temporal changes of the absorption in a spectral range from $-20 \mathrm{meV}$ to $+200 \mathrm{meV}$ relative to the band edge.

Differential transmission spectra (DTS) were taken by modulating the electric field (reverse bias on the p-i-n structure), i.e., $\Delta T / T=\left(T_{\mathrm{E}}-T\right) / T\left(T_{\mathrm{E}}\right.$ is the optical transmission with an electric field, $T$ without a field) with the pump beam always on. This direct comparison allows us to observe the difference in the distribution functions due to the electric field. Typical DTs are shown in figure 1 for an applied field of nominally $16 \mathrm{kV} \mathrm{cm}^{-1}$. At energies above $+40 \mathrm{meV}$ relative to the band edge, it is apparent that at early times $(0,50 \mathrm{fs})$, there are no or small changes in the absorption spectrum because carriers are generated at the bottom of the band. At $150 \mathrm{fs}$, a tail (from $+40 \mathrm{meV}$ up to $+150 \mathrm{meV}$ ) in the DTs is clearly seen; this tail represents a net differential distribution. While there is basically no electron population above the thermal energy of $25 \mathrm{meV}$ when the field is off, the appearance of these high-energy electrons indicates a fast acceleration by the electric field; it occurs in such a short time that only ballistic acceleration can account for it. A simple calculation (energy $=e^{2} E^{2} t^{2} / 2 \mathrm{~m}^{*}$ ) shows that, at $E=16 \mathrm{kV} \mathrm{cm}^{-1}$, a ballistic electron gains $75 \mathrm{meV}$ energy 


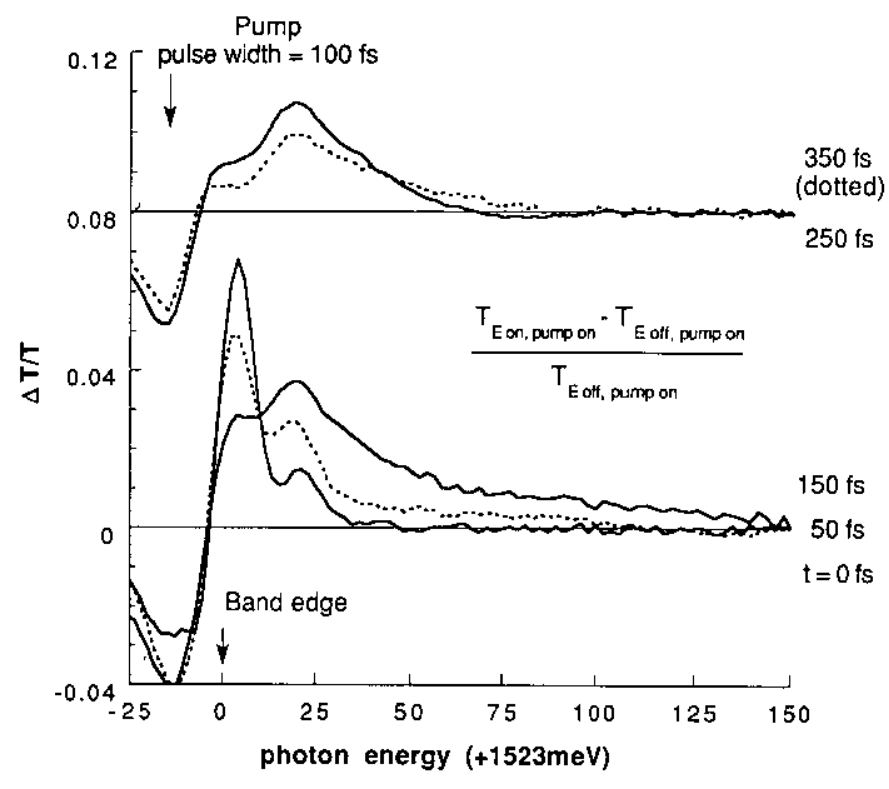

Figure 1. Experimental differential transmission spectra, with the electric field modulated (between 0 and $\left.16 \mathrm{kV} \mathrm{cm}^{-1}\right)$; the pump pulse is always on.

from the field in $150 \mathrm{fs}$. Actually electrons with energies above the band edge as high as $150 \mathrm{meV}$ are observed; these are explained by the finite excitation pulse width $(100 \mathrm{fs})$. Those conduction electrons that are photoinjected during the early part of the optical pulse experience the field acceleration for a time longer than $150 \mathrm{fs}$. At later times ( $250 \mathrm{fs}, 350 \mathrm{fs})$, this high energy tail dccays, indicating an energy overshoot of the electrons confined in the Qw.

In order to determine the electron distribution functions, we reconstructed the transient absorption coeffcients $\alpha(t)$ from DTs (taken modulating both the pump beam and the electric field), with a typical result shown in figure 2. A simple model with three components, namely heavy hole exciton, light hole exciton, and band-to-band continuum absorption, was used to fit $\alpha(t)$ [8]. The distribution functions are obtained directly from the saturation of the band-to-band contribution to $\alpha(t)$. We assumed a Fermi-Dirac distribution near the band edge (in the vicinity of the exciton peaks), but above $40 \mathrm{meV}$ the distribution function could be obtained directly. Good fits to the data were obtained (a representative example is in figure 2 for the zero-field case), indicating that the distribution near the band edge is always thermal; thus carrier-carrier scattering is very strong at these densities $\left(2-3 \times 10^{11} \mathrm{~cm}^{-2}\right)$. At energies above the exciton transitions, we find the distribution is always thermal for the zero-field case. In contrast to the no-field situation [7], with complete exciton-absorption bleaching during the pump pulse and subsequent exciton ionization in $300 \mathrm{fs}$, the presence of the field results in a fast exciton ionization (appearing as a partial recovery of the exciton absorption) occurring almost instantaneously. At $16 \mathrm{kV} \mathrm{cm}^{-1}$ the distribution shows at early times a non-thermal high energy tail, which is due to electrons ballistically accelerated from the band edge, as shown in

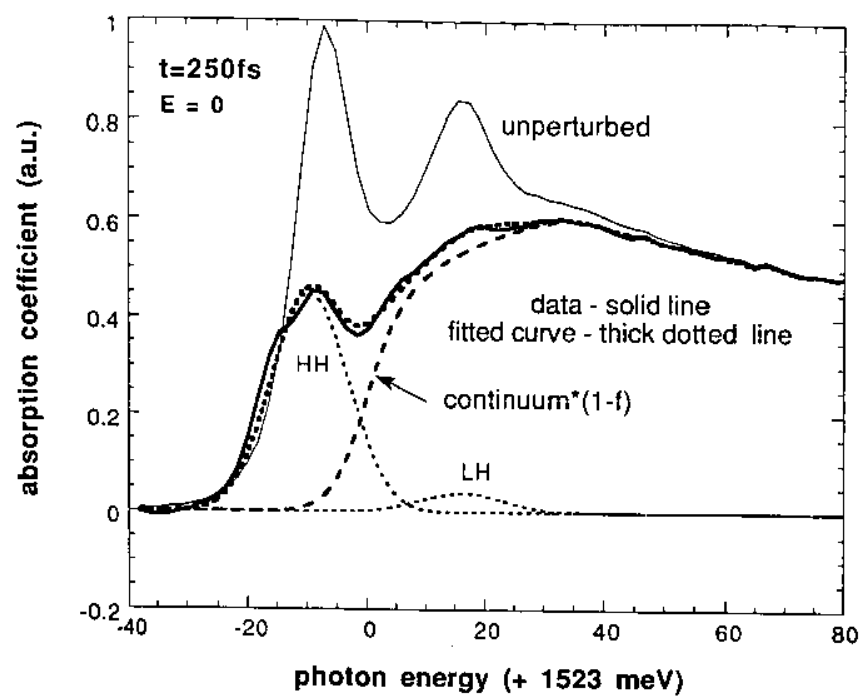

Figure 2. Near-band-edge absorption coefficient for zero electric field, showing the unperturbed spectrum, the spectrum at $250 \mathrm{fs}$ after the pump pulse (thick solid line), and a fit to the spectrum (dotted line), along with the three components of the absorption spectrum as discussed in the text.

figure 3. At times longer than $200 \mathrm{fs}$, the distribution is again thermal, with a temperature several $\mathrm{meV}$ above the zero-field case.

From the distribution functions, we computed the average energy per electron $\left(E_{\mathrm{e}}\right)$ in the $\Gamma$ valley versus time. As shown in figure 4 , for $E=0 \mathrm{~V} \mathrm{~cm}^{-1}$, the initially cold distribution takes about $1.4 \mathrm{ps}$ to reach room temperaturc $(25 \mathrm{mcV})$. The dashed curve is a fit to the expression for heating of the cold plasma by interaction with Lo phonons [9], with an average scattering time of $150 \mathrm{fs}$. This resuit has been obtained in severai experiments, and indicates the accuracy of the fitting procedure.

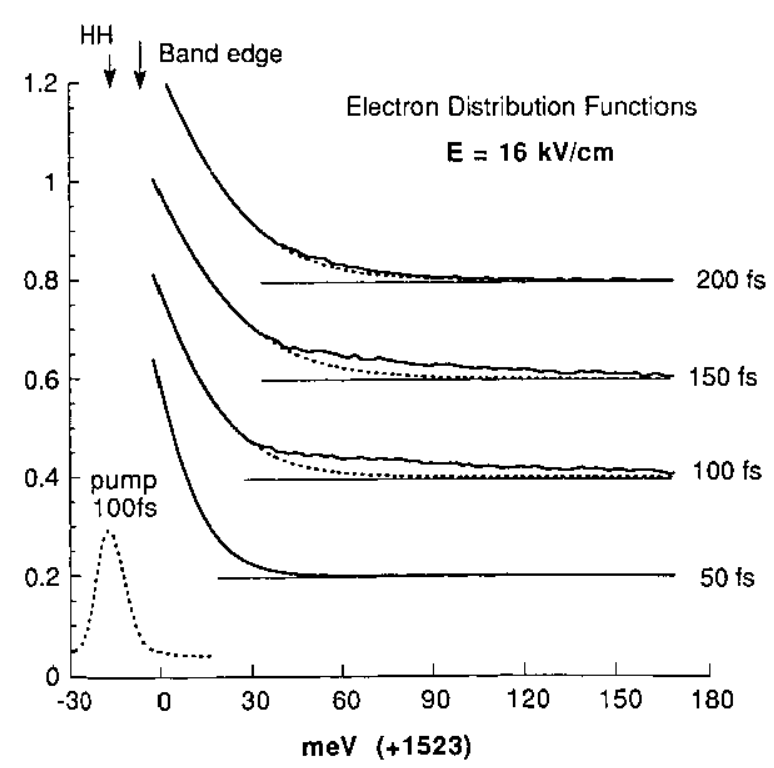

Figure 3. Electron distribution functions for $E=16 \mathrm{kV} \mathrm{cm}^{-1}$ obtained by fitting the transient absorption spectra. 


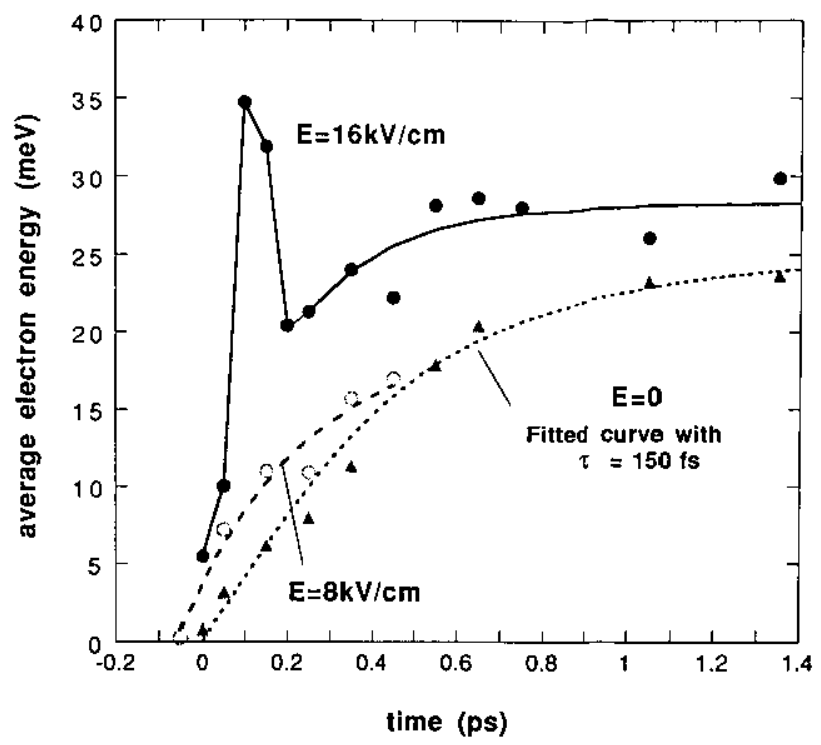

Figure 4. Time-dependent average energy per electron in the quantum well for various electric fields.

For $E=16 \mathrm{kV} \mathrm{cm}^{-1}, E_{\mathrm{e}}$ rapidly accelerates to $35 \mathrm{meV}$ in about $150 \mathrm{fs}$, and corresponds to the growth of the nonthermal high energy tail in the distribution. At this high electric field, $E_{\mathrm{e}}$ shows an overshoot on a $200 \mathrm{fs}$ time scale, with a final average energy only slightly above the zero-field case. There are two contributions to the overshoot. First, the electric field in the gap relaxes rapidly, and will be discussed below. Secondly, high energy electrons can scatter into the continuum states above the barrier (real-space transfer [10]), or at higher energy undergo intervalley scattering, where they no longer contribute to the DTs signal. The low final average energy indicates that the electric field in the gap has relaxed, which is to be expected at the high carrier densities used in this experiment. A measure of the net electric field in the gap is the amplitude of the electroabsorption spectrum on the low energy side of the absorption edge, since the spectrum in this region is more sensitive to the electric field amplitude than to the precise form of the electron distribution function. Hence we have plotted in figure 5 the amplitude of the DTS at 1.505 photon energy. The field is seen to relax on a picosecond time scale, with some intriguing structure occurring on a few hundred fs time scale.

There are two contributions to the field relaxation (switching). First, since the flow of energy from the external bias supply to the gap occurs on a much slower time scale than the transients considered here, all energy gained by the carriers at early times must come from energy stored in the field in the gap region. In fact, the total energy gained by the electrons is comparable to the electromagnetic energy stored in the gap region, thus causing a decrease in the electric field. Secondly, as the electrons and holes move under the influence of the applied field, a dipole is built up which opposes the applied field. This growing dipole will cause the electric field to drop by generating electromagnetic radiation (a well-known property of photoconductive switches [11]),

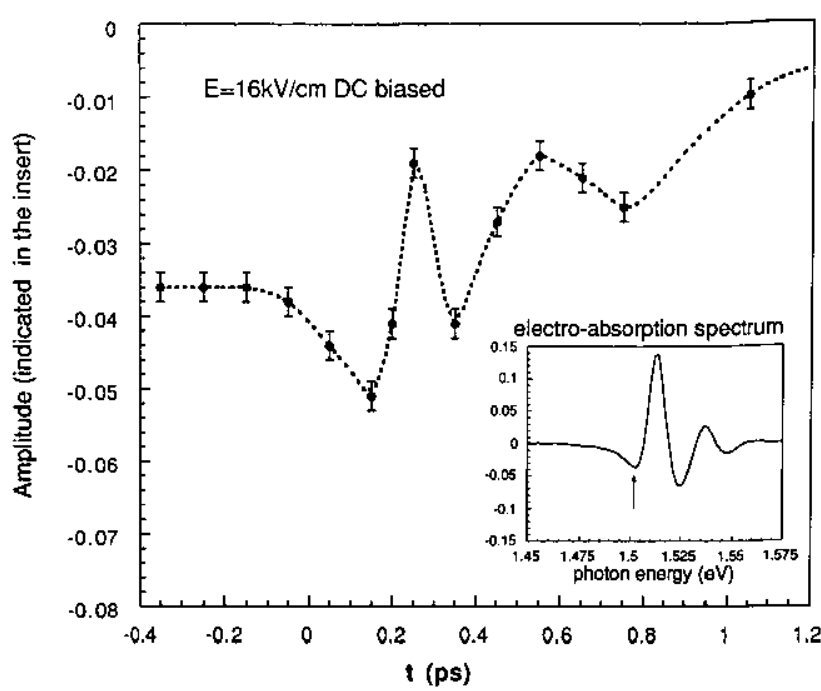

Figure 5. Amplitude of the electroabsorption spectrum (DTS) just below the absorption edge (see inset), which is a measure of the total electric field in the gap.

which may be the source of the structure in figure 5 . The carrier separation eventually will result in a space charge which will screen the applied field. It must be noted that a complete understanding of the carrier dynamics must include their response to the total time-dependent electric field in the gap [12]. The precise dynamics of the electric field, and the relative contributions of the field relaxation and real-space transfer to the energy overshoot are the subject of continuing experimental investigation.

In conclusion, we have used femtosecond spectroscopy to probe high-field parallel transport in quantum wells. We have observed the ballistic acceleration of electrons photoinjected at the band edge on a $150 \mathrm{fs}$ time scale. These experiments enable us to probe directly transient distribution functions occurring during high field transport, including the dynamics of photoconductive switching.

\section{Acknowledgments}

We would like to thank Professors R Grondin, G Mourou and Mr June-Koo Rhee for useful discussions. This work was supported by the AFOSR under grant URI-090-0214.

\section{References}

[1] Shah J 1986 IEEE J. Quantum Electron. QE-22 1728

[2] Bailey D W, Stanton C J and Hess K 1990 Phys. Rev. B 423423

[3] Joshi R P, Grondin R O and Ferry D K 1990 Phys. Rev. B 425685

[4] Levi A F, Hayes J R, Platzman P M and Wiegmann W 1985 Phys. Rev. Lett. 552071

[5] Heiblum M, Nathan M I, Thomas D C and Knoedler C M 1985 Phys. Rev. Lett. 552200 
[6] Duling I N, Norris T, Sizer T, Bado P and Mourou G A 1985 J. Opt. Soc. Am. B 2616

[7] Knox W H, Fork R L, Downer M C, Miller D A B, Chemla D S, Shank C V, Gossard A C and Wiegmann W 1985 Phys. Rev. Lett. 541306

[8] Chemla D S, Miller D A B, Smith P, Gossard A and Wiegmann W 1984 IEEE J. Quantum Electron. QE20265
[9] Rühle W W and Polland H-J 1987 Phys. Rev. B 36 1683

[10] Hess K, Morkoc H, Shichijo H and Streetman B G 1979 Appl. Phys. Lett. 35469

[11] Auston D H, Cheung K P and Smith P R 1984 Appl. Phys. Lett. 45 (3) 284

[12] El-Ghazaly S M, Joshi P R and Grondin R O 1990 IEEE Trans. Microwave Theory Tech. 38629 